\title{
Anatomy and Neurophysiology of Pruritus
}

\author{
Akihiko Ikoma, MD, PhD, Ferda Cevikbas, PhD, Cordula Kempkes, PhD, and \\ Martin Steinhoff, MD, PhD
}

\begin{abstract}
Itch has been described for many years as an unpleasant sensation that evokes the urgent desire to scratch. Studies of the neurobiology, neurophysiology, and cellular biology of itch have gradually been clarifying the mechanism of itch both peripherally and centrally. The discussion has been focused on which nerves and neuroreceptors play major roles in itch induction. The "intensity theory" hypothesizes that signal transduction on the same nerves leads to either pain (high intensity) or itch (low intensity), depending on the signal intensity. The "labeled-line coding theory" hypothesizes the complete separation of pain and itch pathways. Itch sensitization must also be considered in discussions of itch. This review highlights anatomical and functional properties of itch pathways and their relation to understanding itch perception and pruritic diseases.
\end{abstract}

Semin Cutan Med Surg 30:64-70 @ 2011 Elsevier Inc. All rights reserved.

\section{Sensory Nerves and Their Receptors in the Skin}

Tumerous peripheral mediators have been reported as 1 pruritogens. ${ }^{1}$ Histamine, the best-known pruritogen in humans, is released from mast cells when they are activated under various inflammatory conditions, such as the type I allergy. Four histamine receptors $\left(\mathrm{H}_{1}, \mathrm{H}_{2}, \mathrm{H}_{3}\right.$, and $\left.\mathrm{H}_{4}\right)$ have been cloned and characterized. ${ }^{2}$ Histamine-induced neural reactions in the skin, which are axon-reflex flare and itch, seem to be mainly mediated via $\mathrm{H}_{1}$ receptors, because they are all suppressed by application of $\mathrm{H}_{1}$ receptor antagonists. ${ }^{3} \mathrm{H}_{1}$ receptor antagonists are, in fact, effective for some pruritic diseases, such as urticaria, confirming the involvement of histamine and $\mathrm{H}_{1}$ receptors in itch induction in the skin.

Proteases, such as trypsin, tryptase, cathepsins, and kallikreins, also work as pruritogens in inflammatory skin diseases, such as atopic dermatitis. ${ }^{4}$ One of the receptors acti-

Departments of Dermatology and Surgery, University of California, San Francisco, San Francisco, CA.

Dr. Ikoma, Dr. Cevikbas and Dr. Kempkes have nothing to disclose. Dr. Steinhoff has performed consultancy services for which he received compensation for Galderma, Merck, Sanofi-Aventis and Regeneron. On behalf of Dr. Steinhoff, his institution has received financial gifts from Maruho (Japan), and research was supported by ZymoGenetics, L'Oreal and Galderma.

The first two authors contributed equally to this review.

Address reprint requests to A. Ikoma, MD, PhD, and M. Steinhoff, MD, PhD, 513 Parnassus Ave, UCSF Medical Science Building, Room S-1268, San Francisco, CA, 94143-0660. E-mails: IkomaA@derm.ucsf.edu; and SteinhoffM@derm.ucsf.edu vated by these proteases is protease-activated receptor-2 $(\mathrm{PAR})_{2}$. Intracutaneous application of $\mathrm{PAR}_{2}$ agonists induces itch in patients with atopic dermatitis, and $\mathrm{PAR}_{2}$ expression at nerve endings in the skin is also increased in atopic dermatitis, ${ }^{4}$ indicating that proteases induce itch via activation of $\mathrm{PAR}_{2}$ at nerve endings in the skin.

Nevertheless, cutaneous neural receptors, such as H1R or $\mathrm{PAR}_{2}$, which are known to be involved in itch induction, are not always itch-specific. For example, itch is the main sensation induced by histamine when it is applied to the skin by iontophoresis or pricking, whereas pain is the main sensation induced when histamine is injected subcutaneously. ${ }^{5}$ The release of histamine from mast cells in the upper dermis leads to urticaria, which is characterized by the formation of wheal and flare, as well as itch sensation. In contrast, histamine release in the deep dermis or subcutaneous tissue results in a different form of eruption, called "angioedema," which is often accompanied by pain rather than itch. ${ }^{6}$ The sensation caused by skin burn also depends on the depth of the damage. The main sensation is itch when the damage is superficially limited or when the burn is almost healed, but the main sensation is exclusively pain when the damage is deeper.

Two intriguing studies of this difference include one published almost a half century ago, in which a single spicule taken from the pod of an itchy plant known as cowhage (macuna pruriens) induced itch most intensely when it was inserted down to the level of the basal membrane between the epidermis and the dermis. In contrast, itch was never induced when the spicule was inserted to the skin area after the epidermis and upper dermis had 
been removed. ${ }^{7}$ The second study, published in 2005, was performed in healthy human volunteers by the use of transcutaneous electrical stimulation and demonstrated that itch can be induced only when the size of electrodes is very small, suggesting that electrical stimulation restrictedly applied to a superficial layer of the skin is the prerequisite for electrical induction of itch. ${ }^{8}$ Thus, it can be assumed, if the origin of itch is skin, itch is likely induced by an exclusive activation of those nerve endings located in the superficial layer of skin, especially in epidermis and upper dermis around the basal membrane.

However, the origin of pain is not necessarily limited to a deeper level in the skin. Immunohistochemical ultrastructural investigations indicate free nerve endings exist in healthy human epidermis. ${ }^{9}$ Although they elongate into the upper epidermis in many pruritic skin diseases like atopic dermatitis, ${ }^{10}$ a similar type of nerve elongation has also been reported in patients with vulvar vestibulitis accompanied by spontaneous pain and pain sensitization in the genital area. ${ }^{11}$ These studies have led to the hypothesis that nerve endings in the superficial layer of skin are involved in both itch and pain, and the elongated nerves in the epidermis most probably contribute to neural sensitization (Fig. 1)

\section{Primary Afferent Nerves for Itch}

Primary sensory nerves are categorized into 3 groups: $A \beta$, $\mathrm{A} \delta$, and $C$ nerves, depending on their myelination, diameter, and conduction velocity. A $\delta$ and $C$ nerves are mainly involved in the conduction of thermal and pain/itch sensation, whereas $A \beta$ nerves conduct tactile sensation. ${ }^{12}$ Pain sensation originating from the skin surface consists of 2 different subtypes-first pain and second pain and are perceived one after another with a time lag between them. The first pain, conducted by A $\delta$ nerves, is often described as "stabbing," whereas the second one, conducted by $\mathrm{C}$ nerves, is "burning." Itch is more similar to the second "burning" pain than to the first "stabbing" pain, which was one of the reasons why itch was believed to occur by weak activation of pain-conducting C nerves. This led to the "intensity theory," which found consensus for a long time. However, this theory was later contradicted by the findings that morphine reduces pain but simultaneously induces itch and itch evoked by electrical stimulation does not convert into pain even at a higher frequency of stimulation. ${ }^{13}$

The previous human microneurographic studies in which histamine was applied to human skin revealed weak activation in polymodal $\mathrm{C}$ nerves which had a small cutaneous receptive field. ${ }^{14}$ This finding fails to explain histamine-induced intense itch accompanied by a large axon-reflex flare. A subsequent microneurographic study demonstrated histamine-sensitive, mechano-insensitive $\mathrm{C}$ nerves ( $\mathrm{CMi}$ ), whose activation induced by histamine was as long as itch sensation and whose receptive field size is much larger than that of polymodal C-nerves, consistent with a large axon-reflex flare size. ${ }^{15}$ Thus, histamine-induced itch and axon-reflex flare appear to be mediated by CMi nerves.

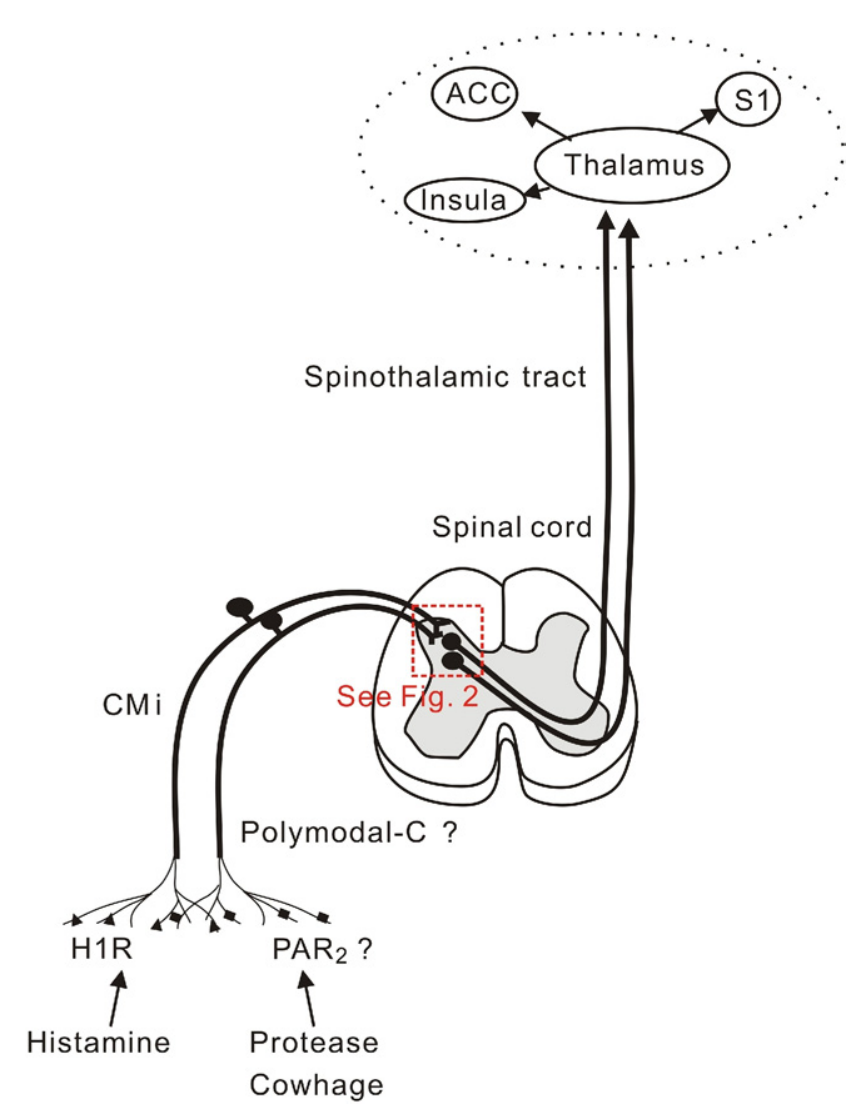

Figure 1 The neural pathway for itch. Histamine activates H1R on the "labeled-line" that is specific to itch, which consists of CMi and spinothalamic tract nerves. Recent studies on itch induced by cowhage, whose protease exerts neural activation via PARs, such as $\mathrm{PAR}_{2}$, indicate the presence of another itch pathway that is composed of polymodal C-nerves and different spinothalamic tract nerves. Both pathways ascend to the thalamus via the dorsal horn in the spinal cord. The activation of several brain areas, including the primary somatosensory cortex (S1), ACC, and insula, seems to be involved in itch perception.

Histamine-sensitive CMi nerves also have other characteristics which differentiate from polymodal $C$ nerves, such as a greater threshold to electrical stimulation, a lower conduction velocity, and a less spontaneous activation, correlating to the specificity theory of itch, i.e., the segregated itch pathway from pain pathways. Thus, the identification of histaminesensitive $C$ nerves in humans supports the "labeled-line coding" of itch, instead of the intensity theory. Some histaminesensitive CMi are also responsive to several pain-inducing substances, such as capsaicin, whose activation is weak. ${ }^{16}$ By contrast, histamine also induces a weak activation of polymodal $\mathrm{C}$ nerves. Thus, histamine-sensitive $\mathrm{CMi}$ might be renamed "itch-selective" rather than "itch-specific" nerves, and substances that intensely activate histamine-sensitive CMi nerves, but not polymodal $\mathrm{C}$ nerves, seem to have pruritogenic potential. ${ }^{17}$

Although the identification of histamine-sensitive CMi was a breakthrough for itch researchers, those nerves are obviously not exclusively for itch. First, most recent studies in rodents provide evidence against the presence of itch-specific 
pathways. Many of the neurons that respond to histamine and other pruritogens are also responsive to noxious stimuli, such as heat, mustard oil, and capsaicin application. ${ }^{18}$ Second, itch without a flare formation is frequently experienced in our daily life, although activation of histamine-sensitive $\mathrm{C}$-nerves causes both itch and axon-reflex flare, which is why histamine-induced itch is always accompanied by axon-reflex flare. One clear example of itch without the accompanying axon-reflex flare is itch induced by cowhage spicules. Desensitization of capsaicin-sensitive skin nerve endings by repeated application of capsaicin inhibits cowhage-induced itch but not histamine-induced itch. ${ }^{19}$ Cowhage has been shown to activate a subpopulation of polymodal C-nerves, but not CMi, which more directly underscores the presence of separate primary afferents for histamine- and cowhageinduced itch. ${ }^{20}$ The presence of multiple subtypes of primary afferents for itch is also supported by the finding that itch is experimentally evoked with weak transepidermal electrical stimulation in healthy volunteers. According to the study, C-nerves that have a much lower threshold to electrical stimulation than histamine-sensitive C-nerves can also mediate itch. ${ }^{8}$ Taken together, these studies indicate there are apparently multiple peripheral neural pathways for itch, some of which are likely to be itch-selective, but others that are not (Fig. 1).

\section{Spinal Receptors for Itch}

No anatomical findings from studies in animals support the presence of itch-specific spinal cord areas. Some studies in rodents have demonstrated neural pathways for itch ascend to the brain via the superficial layer of the dorsal horn, including laminae I and II, in the spinal cord (Fig. 2). 18,21-23 Other studies in cats and primates demonstrate pruritogensensitive superficial dorsal horn neurons. ${ }^{24,25}$ Studies conducted in rats provide data indicating overlaps of itchy stim- uli- and painful stimuli-related areas in the dorsal horn. ${ }^{21,26} \mathrm{~A}$ more recent mouse study has also shown that the pathways for histamine- and nonhistamine-mediated itch overlap those for pain. ${ }^{18}$

Recently, numerous neural receptors in the spinal cord involved in pruritus have been drawing attention as targets of antipruritic targets. ${ }^{27}$ A typical example is opioid receptors. Endogenous opioid peptides, which are endorphins, enkephalins, dynorphins, and endomorphins, exert their effects through 3 classes of receptors: $\mu, \kappa$, and $\delta$. Morphine is one of the best-known $\mu$-opioid receptor (MOR) agonist and has been used as an analgesic agent for centuries. However, the epidural and spinal administration of morphine frequently causes segmental itch as an adverse event. ${ }^{28,29}$ Morphine-induced itch is histamine-independent and antihistamine-resistant ${ }^{30,31}$; mainly occurs in the face, neck, and upper thorax ${ }^{28}$; often spreads rostrally from the injection site ${ }^{32}$; and is inhibited by MOR antagonists. ${ }^{33}$ This suggests that MOR in the spinal cord plays a critical role in controlling itch. Further evidence for this role is that MOR antagonists, such as naloxone and naltrexone, have antipruritic potencies (Fig. 2), not only against morphine-induced itch, but also against dialysis- and cholestasis-related itch that are usually resistant to antihistamine therapies. ${ }^{34-36}$

MOR antagonists reduced itch in some patients with cholestatic pruritus but induced pain at the same time, indicating the role of MOR in controlling the balance between itch and pain. ${ }^{37}$ Meanwhile, $\boldsymbol{\kappa}$-opioid receptor (KOR) agonists can attenuate MOR agonist-induced pruritus without affecting antinociceptive effects of morphine. ${ }^{38}$ Studies in animals have shown that KOR agonists not only suppress morphine-induced itch, but also other subtypes of itch, including both histaminedependent and histamine-independent itch. ${ }^{39}$ In recent clinical studies about the effects of nalfurafine, a newly developed KOR agonist demonstrates the antipuritic roles of KOR, on intractable itch in hemodialysis patients (Fig. 2). ${ }^{40,41}$

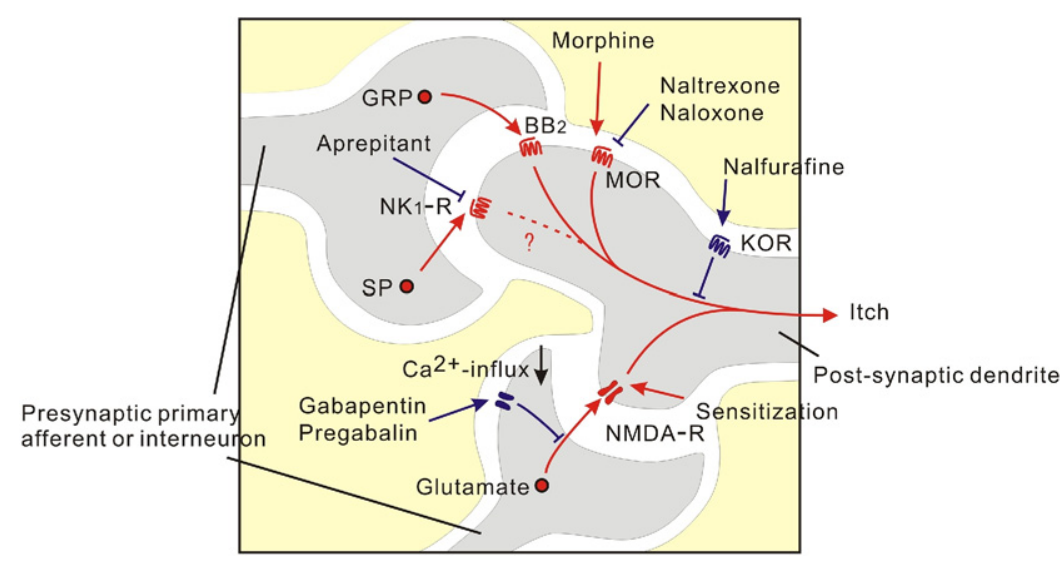

Figure 2 The neurotransmission for itch in the spinal cord. GRP and SP released from presynaptic neurons may exert their pruritogenic roles via BB2 and NK1R, respectively, in the spinal cord. The antipruritic effect of aprepitant, an NKIR antagonist, has been reported. The administration of morphine induces itch via binding to MOR, whereas itch is suppressed by blocking MOR with MOR antagonists, such as naloxone and naltrexone, or activating KOR with KOR agonists, such as nalfurafine. NMDA receptors (NMDA-R) become sensitized by continuous inputs from presynaptic neurons. In such circumstances, glutamate release, which can be blocked by gabapentin- or pregabalin-induced decrease in $\mathrm{Ca}^{2+}$-inflow, leads to more intense activation of NMDA-R, and is supposed to cause more itch. 
Gastrin-releasing peptide (GRP) receptors in the superficial dorsal horn of the spinal cord are also drawing attention as a novel pathway of itch-selective neural transmission. GRP and GRP receptors are broadly expressed in the central nervous system and gastrointestinal tract. GRP plays various physiological roles, such as hormone secretion, blood flow regulation, and smooth muscle contraction via the activation of GRP receptors, which are also called bombesin receptor-2 (BB2). ${ }^{42,43}$ Recent studies have added itch transduction to the functions of GRP and GRP receptors ${ }^{44,45}$ : BB2-mutant mice have normal pain perception but show less scratching behavior when pruritogens are applied. In wild-type mice, however, the intrathecal application of BB2 antagonists reduces scratching behavior induced by those pruritogens. ${ }^{44}$ Moreover, the ablation of lamina I neurons expressing BB2 results in lack of scratching responses to pruritogens. ${ }^{45}$ Thus, GRP receptors might be exclusively expressed in itch-specific nerves. In dorsal root ganglion neurons, GRP is specifically distributed in a small subset of small-to medium-sized neurons and colocalizes calcitonin gene-related peptides and peripherin, a marker for unmyelinated $C$ nerves. In the dorsal horn of the spinal cord, GRP is found in the lamina I, and the outer layer of the lamina II. ${ }^{44}$

Substance P (SP) is a neuropeptide of the tachykinin family. Neurokinin-1 receptor (NK1R), broadly expressed in the peripheral and central nervous system, has the greatest affinity for SP. ${ }^{46}$ Several studies in rodents have demonstrated a high expression of NK1R in the superficial dorsal horn of the spinal cord, including the lamina I. ${ }^{47-52}$ The ablation of NK1R-expressing dorsal horn neurons reduces hyperalgesia in pain models ${ }^{53}$ suggesting SP and NK1R are involved in pain transmission at the spinal cord level. The antipruritic effect of aprepitant, an NK1R antagonist which has affinities for NK2R and NK3R to some extent, has been reported in studies of patients with Sézary syndrome ${ }^{54}$ and various types of chronic pruritus. ${ }^{55}$ The antipruritic potential of NK1R antagonists, however, remains to be determined by large-scale clinical studies.

\section{Secondary Afferent Nerves for Itch}

Histamine-sensitive neurons of cats were found in a small subgroup of the lamina I neurons whose axons ascend to the contralateral thalamus through the spinothalamic tract (STT) ${ }^{24}$ A later study of rats with dry skin found itch-related c-fos expression in lamina I but not in lamina II or III, findings that differ from the capsaicin-induced c-fos expression that can be observed throughout laminae I, II, and III. ${ }^{21}$ Similar to histamine-sensitive C-nerves in humans, histamine-sensitive STT tract neurons in cats do not respond to mechanical or thermal stimulation and have a slow conduction velocity. Furthermore, they project mainly to the ventral, posterior, inferior nucleus, and the ventral periphery of the ventral, posterior, lateral nucleus of the lateral thalamus, whereas nociceptive STT neurons project mainly to the nucleus submedius of the medial thalamus. These findings indicate the labeled-line coding of itch in the secondary afferents is just like that in the primary afferents. This similarity is also supported by the aforementioned recent findings on GRP and GRP receptors in the spinal cord.

By contrast, a recent study in primates revealed histamine-responding STT neurons have a wide dynamic range and also respond to capsaicin, although they are likely to contribute more to pain than to itch. ${ }^{56}$ Another primate study investigating STT neurons found that no STT neurons are reactive to both histamine and cowhage; they only respond to either histamine or cowhage, suggesting separate STT pathways for histamine- and cowhage-induced itch. $^{57}$

\section{Brain Functions for Itch}

Studies of itch-related brain functions are gradually increasing because of technological advances in functional brain imaging and earlier studies on pain-related brain functions. Pain-related brain imaging studies have so far reached the general consensus that the spatial, temporal and intensity aspects of pain perception are processed in the primary (S1) and secondary somatosensory cortex (S2), whereas the affective and motivational aspects are in the anterior cingulate cortex (ACC) and the insular cortex. ${ }^{58}$ The thalamus, prefrontal cortex, premotor areas, and cerebellum are also activated during pain perception. ${ }^{59,60}$ Itch-related brain imaging studies using positron emission tomography have shown the prefrontal cortex, premotor areas, such as S1 and ACC, are activated during itch perception. ${ }^{61,62}$ Coactivation of ipsilateral premotor areas might reflect the desire to scratch. ${ }^{63}$ Both ascending and descending pathways to control itch have been investigated with functional brain imaging techniques. A positron emission tomography study demonstrated the midbrain periaquaductal gray is activated to reduce itch during simultaneous itch and pain stimuli, but not during either itch or pain stimuli alone. ${ }^{64} \mathrm{~A}$ functional magnetic resonance imaging study revealed scratching of the skin activates the putamen only when itch coexists. ${ }^{65}$ Generally speaking, however, pain- and itch-related brain activation overlap. It remains unclear whether any brain area or brain activation pattern is specific to itch. One obstacle for brain imaging studies of itch is the difficulty in standardizing itchy stimuli because the duration and intensity of itch is by nature hard to control. The hope is this problem will be overcome by newly developed experimental methods to evoke itch, such as electrically evoked itch. ${ }^{8}$

\section{Modification of Itch Pathways in Diseases: Itch Sensitization}

It is well known that patients with chronic pain, such as postherpetic neuralgia, not only complain of spontaneous pain but also pain sensitization. Pain sensitization causes pain to be evoked by weak mechanical stimuli, such as warm 
water, which usually are not painful. Patients also feel strong pain from light pin-pricking stimuli to the skin. These phenomena, called allodynia and pin-prick hyperalgesia, respectively, occur because of neural sensitization, i.e., lowering of neural thresholds for external stimuli. ${ }^{66} \mathrm{Com}-$ parable symptoms are observed in patients with chronic pruritus. Patients with atopic dermatitis, for example, not only feel spontaneous itch, but also feel itchy when weak mechanical stimuli, such as contact of wool fibers are applied to the skin. An experimental study in patients with atopic dermatitis showed intracutaneous application of histamine induces much more intense itch in the eczematous skin as compared with their noneczematous skin or the completely intact skin of healthy volunteers. ${ }^{67}$ These phenomena, parallel to allodynia and hyperalgesia, are called alloknesis and hyperknesis. The similarity between allodynia and alloknesis, as well as between hyperalgesia and hyperknesis, indicates similar mechanisms are likely to be involved in both itch and pain sensitization.

A decent amount of evidence has demonstrated that central sensitization plays a crucial role in pain sensitization, ${ }^{67}$ whereas few studies regarding itch sensitization have been reported. The postsynaptic N-methyl-D-aspartate (NMDA) receptor, which glutamate binds to, is one of the receptors known to be involved in pain via induction of central sensitization at the spinal level. Binding of glutamate usually is not strong enough to activate NMDA receptors because of $\mathrm{Mg}^{2+}$ blockade. However, continuous activation of peripheral nociceptive afferents under various conditions, such as injury and inflammation, leads to depolarization in the postsynaptic cells of the spinal cord, which then removes the $\mathrm{Mg}^{2+}$ blockade from NMDA receptors, enabling them to be activated by glutamate binding. The activation of NMDA receptors leads to $\mathrm{Ca}^{2+}$ influx and intracellular signal transduction cascades, which results in the phosphorylation of ion channels, and subsequently in the excitatory postsynaptic potential. Altogether, NMDA receptors play a significant role in prolonged and increased excitation of postsynaptic neuronal excitability-in other words, central sensitization. ${ }^{42}$

As the result of central sensitization, signals from primary afferents for pain or itch cause more intense activation of postsynaptic spinal neurons for pain or itch than they usually do, which explains hyperalgesia (or hyperknesis). Moreover, not only signals from pain (or itch) nerves, but also those from others, such as A- $\beta$ nerves, whose activation usually induces tactile sensation, activate postsynaptic spinal neurons for pain or itch, which is the explanation for allodynia (or alloknesis). Thus, central sensitization caused by diseases multiplies pathways for pain and itch. In pruritic diseases, such as atopic dermatitis, one consequence is itch is evoked by those stimuli which normally induce pain and inhibit itch. ${ }^{68}$ Moreover, inflammatory mediators, such as bradykinin, which are known to be algogens, can also induce itch under itch sensitization, ${ }^{69}$ which emphasizes central sensitization should be considered for therapeutic strategies.

\section{Summary and Future Aspects}

Recent findings indicate itch is a complex process, including various cells, mediators and receptors in the peripheral skin, spinal cord, and brain. Molecular as well as pharmacologic studies in which the authors used null mice have enhanced our understanding about the role of histamine-independent itch pathways, including GRP, $\mathrm{PAR}_{2}, \mathrm{NK} 1 \mathrm{R}$, and others. The "intensity theory" and the "labeled-line coding theory" requires further research to fully understand the complex regulation of itch, which also includes interneurons (Fig. 2) and probably various areas and receptors in the brain. Understanding the circuits of neuronal communication between keratinocytes or immune cells and nerve endings will result in improved therapies against chronic itch in inflammatory or systemic skin diseases. The current challenge of itch research is to clarify molecular mechanisms of itch perception, which plays a major role in developing appropriate treatments against pruritus. Meeting this challenge appears to require further investigation of the differences, similarities, and crosstalk between itch and pain, to understand the crosstalk between nerves and skin cells or immune cells and to improve our knowledge about central sensitization and how the spinal cord and brain are dysregulated during chronic itch.

\section{References}

1. Ikoma A, Steinhoff M, Ständer S, et al: The neurobiology of itch. Nat Rev Neurosci 7:535-547, 2006

2. Jutel M, Akdis M, Akdis CA: Histamine, histamine receptors and their role in immune pathology. Clin Exp Allergy 39:1786-1800, 2009

3. Greaves MW, Davies MG: Histamine receptors in human skin: Indirect evidence. Br J Dermatol 107 suppl 23:101-105, 1982

4. Steinhoff M, Neisius U, Ikoma A, et al: Proteinase-activated receptor-2 mediates itch: A novel pathway for pruritus in human skin. J Neurosci 23:6176-6180, 2003

5. Rosenthal SR: Histamine as the chemical mediator for cutaneous pain. J Invest Dermatol 69:98-105, 1977

6. Black AK: Unusual urticarias. J Dermatol 28:632-634, 2001

7. Shelley WB, Arthur RP: Mucunain, the active pruritogenic proteinase of cowhage. Science 122:469-470, 1955

8. Ikoma A, Handwerker H, Miyachi Y, et al: Electrically evoked itch in humans. Pain 113:148-154, 2005

9. Hilliges M, Wang L, Johansson O: Ultrastructural evidence for nerve fibers within all vital layers of the human epidermis. J Invest Dermatol 104:134-137, 1995

10. Urashima R, Mihara M: Cutaneous nerves in atopic dermatitis. A histological, immunohistochemical and electron microscopic study. Virchows Arch 432:363-370, 1998

11. Bohm-Starke N, Hilliges M, Falconer C, et al: Increased intraepithelial innervation in women with vulvar vestibulitis syndrome. Gynecol Obstet Invest 46:256-260, 1998

12. Lawson SN: Phenotype and function of somatic primary afferent nociceptive neurones with C-, Adelta- or Aalpha/beta-fibres. Exp Physiol $87: 239-244,2002$

13. Tuckett RP: Itch evoked by electrical stimulation of the skin. J Invest Dermatol 79:368-373, 1982

14. Handwerker HO, Forster C, Kirchhoff C: Discharge patterns of human C-fibers induced by itching and burning stimuli. J Neurophysiol 66 : 307-315, 1991 
15. Schmelz M, Schmidt R, Bickel A, et al: Specific C-receptors for itch in human skin. J Neurosci 17:8003-8008, 1997

16. Schmelz M, Schmidt R, Weidner C, et al: Chemical response pattern of different classes of C-nociceptors to pruritogens and algogens. J Neurophysiol 89:2441-2448, 2003

17. Schmelz M: Itch—mediators and mechanisms. J Dermatol Sci 28:9196, 2002

18. Akiyama T, Carstens MI, Carstens E: Excitation of mouse superficial dorsal horn neurons by histamine and/or PAR-2 agonist: Potential role in itch. J Neurophysiol 102:2176-2183, 2009

19. Johanek LM, Meyer RA, Hartke T, et al: Psychophysical and physiological evidence for parallel afferent pathways mediating the sensation of itch. J Neurosci 27:7490-7497, 2007

20. Namer B, Carr R, Johanek LM, et al: Separate peripheral pathways for pruritus in man. J Neurophysiol 100:2062-2069, 2008

21. Nojima H, Carstens MI, Carstens E: c-fos expression in superficial dorsal horn of cervical spinal cord associated with spontaneous scratching in rats with dry skin. Neurosci Lett 347:62-64, 2003

22. Akiyama T, Merrill AW, Carstens MI, et al: Activation of superficial dorsal horn neurons in the mouse by a PAR-2 agonist and 5-HT: Potential role in itch. J Neurosci 29:6691-6699, 2009

23. Nakano T, Andoh T, Lee JB, et al: Different dorsal horn neurons responding to histamine and allergic itch stimuli. Neuroreport 19:723726, 2008

24. Andrew D, Craig AD: Spinothalamic lamina I neurons selectively sensitive to histamine: A central neural pathway for itch. Nat Neurosci 4:72-77, 2001

25. Davidson S, Giesler GJ: The multiple pathways for itch and their interactions with pain. Trends Neurosci 33:550-558, 2010

26. Jinks SL, Carstens E: Responses of superficial dorsal horn neurons to intradermal serotonin and other irritants: Comparison with scratching behavior. J Neurophysiol 87:1280-1289, 2002

27. Cevikbas F, Steinhoff M, Ikoma A: Role of spinal neurotransmitter receptors in itch: new insights into therapies and drug development. CNS Neurosci Ther. 2010. [Epub ahead of print]. doi: 10.1111/j.17555949.2010.00201.x.

28. Ballantyne JC, Loach AB, Carr DB: Itching after epidural and spinal opiates. Pain 33:149-160, 1988

29. Mikuni I, Hirai H, Toyama Y, et al: Efficacy of intrathecal morphine with epidural ropivacaine infusion for postcesarean analgesia. J Clin Anesth 22:268-273, 2010

30. Krause L, Shuster S: Mechanism of action of antipruritic drugs. Br Med J (Clin Res Ed) 287:1199-1200, 1983

31. Rosow CE, Moss J, Philbin DM, et al: Histamine release during morphine and fentanyl anesthesia. Anesthesiology 56:93-96, 1982

32. Bromage PR, Camporesi EM, Durant PA, et al: Rostral spread of epidural morphine. Anesthesiology 56:431-436, 1982

33. Saiah M, Borgeat A, Wilder-Smith $\mathrm{OH}$, et al: Epidural-morphine-induced pruritus: Propofol versus naloxone. Anesth Analg 78:11101113,1994

34. Bergasa NV: The pruritus of cholestasis. J Hepatol 43:1078-1088, 2005

35. Peer G, Kivity S, Agami O, et al: Randomised crossover trial of naltrexone in uraemic pruritus. Lancet 348:1552-1554, 1996

36. Phan NQ, Bernhard JD, Luger TA, et al: Antipruritic treatment with systemic $\mu$-opioid receptor antagonists: A review. J Am Acad Dermatol 63:680, 2010

37. McRae CA, Prince MI, Hudson M, et al: Pain as a complication of use of opiate antagonists for symptom control in cholestasis. Gastroenterology 125:591-596, 2003

38. Lee H, Naughton NN, Woods JH, et al: Effects of butorphanol on morphine-induced itch and analgesia in primates. Anesthesiology 107: 478-485, 2007

39. Togashi Y, Umeuchi H, Okano K, et al: Antipruritic activity of the kappa-opioid receptor agonist, TRK-820. Eur J Pharmacol 435:259264, 2002

40. Kumagai H, Ebata T, Takamori K, et al: Effect of a novel kappa-receptor agonist, nalfurafine hydrochloride, on severe itch in 337 haemodialysis patients: A phase III, randomized, double-blind, placebo-controlled study. Nephrol Dial Transplant 25:1251-1257, 2010
41. Wikstrom B, Gellert R, Ladefoged SD, et al: Kappa-opioid system in uremic pruritus: Multicenter, randomized, double-blind, placebo-controlled clinical studies. J Am Soc Nephrol 16:3742-3747, 2005

42. Cornelio DB, Roesler R, Schwartsmann G: Gastrin-releasing peptide receptor as a molecular target in experimental anticancer therapy. Ann Oncol 18:1457-1466, 2007

43. Ischia J, Patel O, Shulkes A, et al: Gastrin-releasing peptide: Different forms, different functions. Biofactors 35:69-75, 2009

44. Sun YG, Chen ZF: A gastrin-releasing peptide receptor mediates the itch sensation in the spinal cord. Nature 448:700-703, 2007

45. Sun YG, Zhao ZQ, Meng XL, et al: Cellular basis of itch sensation. Science 325:1531-1534, 2009

46. Roosterman D, Goerge T, Schneider SW, et al: Neuronal control of skin function: The skin as a neuroimmunoendocrine organ. Physiol Rev 86:1309-1379, 2006

47. Bleazard L, Hill RG, Morris R: The correlation between the distribution of the NKl receptor and the actions of tachykinin agonists in the dorsal horn of the rat indicates that substance $\mathrm{P}$ does not have a functional role on substantia gelatinosa (lamina II) neurons. J Neurosci 14:7655-7664, 1994

48. Brown JL, Liu H, Maggio JE, et al: Morphological characterization of substance P receptor-immunoreactive neurons in the rat spinal cord and trigeminal nucleus caudalis. J Comp Neurol 356:327-344, 1995

49. Costa SK, Starr A, Hyslop S, et al: How important are NKl receptors for influencing microvascular inflammation and itch in the skin? Studies using Phoneutria nigriventer venom. Vascul Pharmacol 45:209-214, 2006

50. Littlewood NK, Todd AJ, Spike RC, et al: The types of neuron in spinal dorsal horn which possess neurokinin-1 receptors. Neuroscience 66: 597-608, 1995

51. Liu H, Brown JL, Jasmin L, et al: Synaptic relationship between substance $\mathrm{P}$ and the substance $\mathrm{P}$ receptor: Light and electron microscopic characterization of the mismatch between neuropeptides and their receptors. Proc Natl Acad Sci U S A 91:1009-1013, 1994

52. Nakaya Y, Kaneko T, Shigemoto R, et al: Immunohistochemical localization of substance $\mathrm{P}$ receptor in the central nervous system of the adult rat. J Comp Neurol 347:249-274, 1994

53. Mantyh PW, Rogers SD, Honore P, et al: Inhibition of hyperalgesia by ablation of lamina I spinal neurons expressing the substance P receptor. Science 278:275-279, 1997

54. Duval A, Dubertret L: Aprepitant as an antipruritic agent? N Engl J Med 361:1415-1416, 2009

55. Ständer S, Siepmann D, Herrgott I, et al: Targeting the neurokinin receptor 1 with aprepitant: A novel antipruritic strategy. PLoS ONE 5:e10968, 2010

56. Simone DA, Zhang X, Li J, et al: Comparison of responses of primate spinothalamic tract neurons to pruritic and algogenic stimuli. J Neurophysiol 91:213-222, 2004

57. Davidson S, Zhang X, Yoon $\mathrm{CH}$, et al: The itch-producing agents histamine and cowhage activate separate populations of primate spinothalamic tract neurons. J Neurosci 27:10007-10014, 2007

58. Vogt BA: Pain and emotion interactions in subregions of the cingulate gyrus. Nat Rev Neurosci 6:533-544, 2005

59. Kwan CL, Crawley AP, Mikulis DJ, et al: An fMRI study of the anterior cingulate cortex and surrounding medial wall activations evoked by noxious cutaneous heat and cold stimuli. Pain 85:359-374, 2000

60. Moulton EA, Keaser ML, Gullapalli RP, et al: Regional intensive and temporal patterns of functional MRI activation distinguishing noxious and innocuous contact heat. J Neurophysiol 93:2183-2193, 2005

61. Darsow U, Drzezga A, Frisch M, et al: Processing of histamine-induced itch in the human cerebral cortex: A correlation analysis with dermal reactions. J Invest Dermatol 115:1029-1033, 2000

62. Drzezga A, Darsow U, Treede RD, et al: Central activation by histamineinduced itch: Analogies to pain processing: A correlational analysis of O-15 H2O positron emission tomography studies. Pain 92:295-305, 2001

63. Hsieh JC, Hägermark O, Ståhle-Bäckdahl M, et al: Urge to scratch represented in the human cerebral cortex during itch. J Neurophysiol 72:3004-3008, 1994 
64. Mochizuki H, Tashiro M, Kano M, et al: Imaging of central itch modulation in the human brain using positron emission tomography. Pain 105:339-346, 2003

65. Vierow V, Fukuoka M, Ikoma A, et al: Cerebral representation of the relief of itch by scratching. J Neurophysiol 102:3216-3224, 2009

66. Kuner R: Central mechanisms of pathological pain. Nat Med 16:12581266,2010

67. Ikoma A, Rukwied R, Ständer S, et al: Neuronal sensitization for hista- mine-induced itch in lesional skin of patients with atopic dermatitis. Arch Dermatol 139:1455-1458, 2003

68. Ikoma A, Fartasch M, Heyer G, et al: Painful stimuli evoke itch in patients with chronic pruritus: Central sensitization for itch. Neurology 62:212-217, 2004

69. Hosogi M, Schmelz M, Miyachi Y, et al: Bradykinin is a potent pruritogen in atopic dermatitis: A switch from pain to itch. Pain 126:16-23, 2006 